\title{
MULTI-RESOLUTION FEDERATIONS IN SUPPORT OF OPERATIONAL AND HIGHER LEVEL COMBINED/JOINT COMPUTER ASSISTED EXERCISES
}

\author{
Erdal Cayirci \\ CAX Support Branch / Elect Eng \& Comp Sci Dep \\ NATO Joint Warfare Center / University of Stavanger \\ Stavanger, NORWAY
}

\begin{abstract}
Multi-resolution military federations are still in their infancy. Their usefulness are often discussed and there is a confusion about the related taxonomy. In this paper, first the definitions for the field are clarified, and the latest developments are explained. Computer assisted exercise architectures and the place of constructive simulations in this architecture are also explained. Then the advantages of multi-resolution federations in support of computer assisted exercises are elaborated by using an example vignette.
\end{abstract}

\section{INTRODUCTION}

In the military, simulation is used for various purposes, such as, capability development, defense planning, operation support and training. Simulation justifies itself, perhaps the best for military, because it reduces the cost of these functions considerably. Simulation is also the only way to test and to train for some special situations, such as nuclear, biological and chemical contamination, and operations that require large scale mobilization and movement. Creating and maintaining such situations in real life are often impractical. There is also casualty risk in military live training and experimentation. Therefore, simulation is not only a cost effective approach for military but also the only practical and safe way for testing and training in special and hazardous environments. Therefore, military simulation systems (Cayirci 2009, Surdu 2000) are used extensively. They can be categorized into three broad classes shown in Table 1 (Cayirci 2006):

Table 1: Military simulations

\begin{tabular}{|l|l|l|}
\hline Category & People & Systems \\
\hline Live & Real & Real \\
\hline Virtual & Real & Simulated \\
\hline Constructive & Simulated & Simulated \\
\hline
\end{tabular}

- Live simulation refers to a simulation that involves real people operating real systems. For example two pilots can be trained for dog fighting by using real aircrafts in the air. In this case the aircrafts and the pilots are real but the interactions between the aircrafts are simulated and the simulation decides how effective the pilots and the aircrafts against each other. Similarly, all the weapon systems can be equipped with emitters, and all the equipment and personnel can be equipped with sensors. If the weapons are aimed and fired correctly, the emission by the emitters can be sensed by the sensors, which indicates a hit and a kill based on some stochastic processes.

- Virtual simulation refers to a simulation that involves real people operating simulated systems. Examples for this are aircraft and tank simulators, where a simulator but not a real system is used to train a pilot or tank crew.

- Constructive simulation refers to a simulation that involves simulated people operating in simulated environments. Combat models that compute the possible outcomes of the decisions taken by headquarters fall in this category. In these simulations people and unit are also simulated, as well as, combat systems and environment.

Although the interaction of these categories of simulation systems is possible by using the state of the art technology, and all of them, i.e., live, virtual and constructive simulations, may be used during the execution of a computer assisted exercise (CAX), often a military constructive simulation system constitutes the core of a CAX. Constructive simulations are designed 
to find out the possible outcomes of the courses of actions taken by the real people. They are constructed by many models often stochastic processes that calculate the results of interactions between the entities or units in a theatre. Constructive simulation systems can be classified into two categories according to their resolution as summarized in Table 2:

Table 2: Military constructive simulations

\begin{tabular}{|c|c|c|c|}
\hline Category & Level & Objects & Terrain \\
\hline $\begin{array}{l}\text { High } \\
\text { Resolution }\end{array}$ & Entity & $\begin{array}{l}\text { Singular } \\
\text { objects, e.g. a } \\
\text { tank, a troop }\end{array}$ & $\begin{array}{l}\text { High } \\
\text { resolution, } \\
200 \times 200 \mathrm{~km}\end{array}$ \\
\hline $\begin{array}{l}\text { Highly } \\
\text { Aggregated }\end{array}$ & Aggregate & $\begin{array}{ll}\text { Units, e.g. a } \\
\text { battalion, } \\
\text { company }\end{array}$ & $\begin{array}{l}\text { Low } \\
\text { resolution, } \\
4000 \times 4000 \\
\mathrm{~km}\end{array}$ \\
\hline
\end{tabular}

- High resolution simulations are entity level simulations where singular military objects, e.g. soldiers, tanks, aircrafts, are the primary objects represented. The resolution of terrain data is high sometimes up to the plans of individual buildings. However, the simulated terrain is often limited to $200 \mathrm{~km} \times 200 \mathrm{~km}$. High resolution simulations better suit for tactical level. However, they are not only for tactical level simulation. They provide higher resolution that may be required also for operational and higher level purposes. Therefore, high resolution simulation systems should not be called as tactical simulations.

- Highly Aggregated simulations are aggregate level simulations where collections of military assets, i.e., units, are the primary objects represented. They use lower resolution terrain data but they can simulate in very large areas as large as continents. Similar to high resolution simulations, there is a tendency to call highly aggregated simulations as operational level simulations, which is not correct. Aggregate level simulations may be very useful also for tactical purposes. Please note that in civilian content tactical level is over operational level, which is opposite to the military hierarchy. In this paper we use the military hierarchy in our definitions.

State of the art high resolution simulations can be used for simulating operations in regions as large as $2500 \mathrm{~km} \times 2500$ $\mathrm{km}$. On the other hand aggregate level simulations tend to be capable to simulate the entities such as a single troop and a tank.

The constructive simulation systems can also be categorized based on their functionalities as follows:

- Service models are the simulation systems developed for the needs of a single service, i.e., army, navy or air force.

- Joint models are either the simulation systems that fulfill the requirements of all services or federations made up of service models.

- Expert models are developed specifically to simulate certain functionalities such as logistics, intelligence, electronic warfare, homeland security and space operations.

In this paper we examine the usefulness and practicality of multi-resolution federations (Bowers 2003) that integrate all these classes of constructive simulations, as well as, live and virtual simulations into a seamless simulation environment through distributed simulation technologies, mainly high level architecture (HLA) (IEEE 2000a, IEEE 2000b, IEEE 2000c, Defense 1998). We aim:

- To provide a tutorial on CAX, and how computer simulations are used in a CAX

- To provide a tutorial on multi resolution constructive simulation federations, which is an emerging technology for the military application of distributed computer simulations

- To explain the importance of multi resolution federations for combined/joint exercises in operational and higher levels.

In Section 2 we elaborate a CAX architecture, which is typically made up of two main components, training audience (TA) and exercise control (EXCON). In the same section we explain how constructive simulation systems are used in a CAX. In Section 3 multi resolution federations and NATO Training Federation (NTF) are introduced. Then an example for incidents that require high resolution modeling in operational and higher level exercises is given in Section 4 . We conclude our paper in Section 5.

\section{TRAINING AUDIENCE, EXERCISE CONTROL, RESPONSE CELLS AND SIMULATION}

A CAX is set up based on TA and training objectives (Cayirci 2009, Cayirci 2007a). Throughout CAX process from the start of exercise specification until the end of after action review (AAR) these two should always be the main focus. A TA can be 
single level, multi-level, cross level and both cross and multi level as shown in Figure 1. Multi level training audience represent multiple levels of command trained at the same time in the context of a single scenario. Cross level TA includes units or headquarters at the same level of command. When the units in a cross level TA are from different services, the exercise becomes joint. A TA can have forces from different nations, which makes the exercise combined. In more and more exercises civilian national/international agencies and organizations like police, fire department, health agencies and UN are involved in. Most of the time these civilian organizations become a part of EXCON and constitute white or gray cell as shown in Figure 3. They may also be a part of TA.

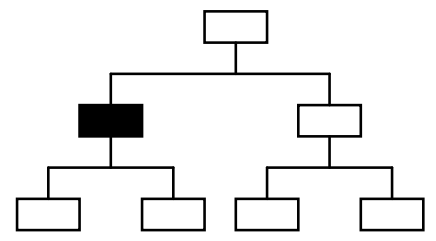

a. Single level

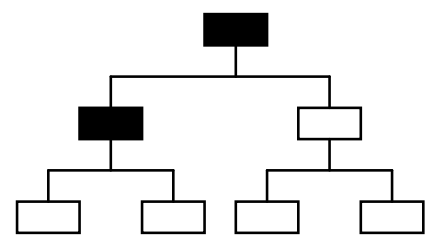

c. Multi level

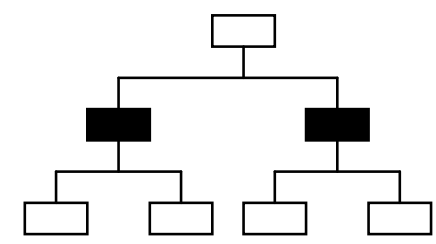

b. Cross level

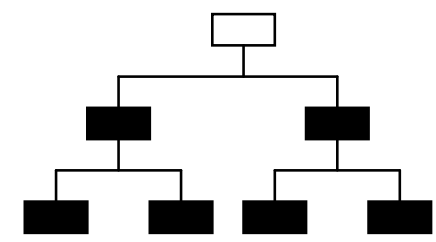

b. Multi and Cross level

Figure 1: Training audience

TA can be co-located or various parts of TA can be located in geographically remote sites, i.e., different cities, countries, continents, etc. The exercises that have TA components located remote sites are called distributed exercises. Please note that distributed simulation and distributed exercise are different things. A distributed exercise can be supported by a centralized simulation system or a centralized exercise can be supported by a distributed simulation. Locating client workstations of a simulation in remote sites does not make a simulation distributed. In distributed simulation computation for the simulations are carried out by multiple processes often in remote machines.

The second component of a CAX is EXCON. A typical EXCON model is shown in Figure 2. Training team (TT) consists of mentors, observer/trainers $(\mathrm{O} / \mathrm{T})$, subject mater experts (SME) and analysts. TT is deployed with TA, observe TA, provide on site instructions and training, and collects inputs for AAR and the evaluation of TA. Exercise center (EXCEN) is the organization responsible for the consistent and coherent flow of the exercise according to the exercise objectives. EXCEN is explained in detail below. Experimentation team runs the experiments planned in conjunction with the exercise. Finally support team has the elements like real life support (RLS), visitor officer bureau (VOB), public information centre (PIC), security office and computers/communications support team.

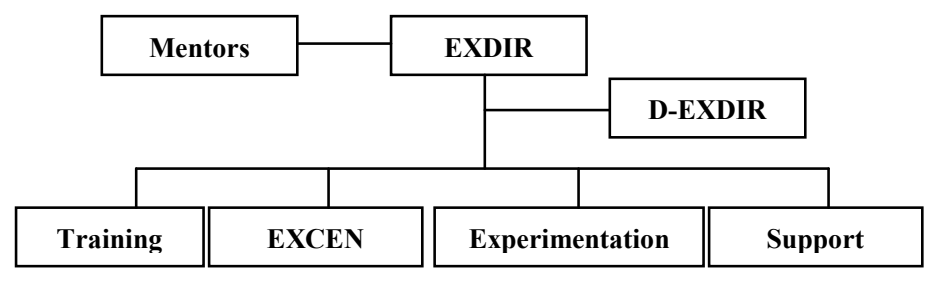

Figure 2: EXCON structure

EXCEN functions can be categorized into five broad classes as situation centre (SITCEN), higher control (HICON), lower control (LOCON), white/gray cell and situation forces (SITFOR). SITCEN monitors the current status of the exercise closely and steers it according to the training objectives. HICON and LOCON represent the command levels/echelons that would normally be at the level above and below the TA respectively. White/gray cell is a response cell that is composed of subject matter experts (SME) or role players representing agencies, organizations, institutions and individuals outside of the 
own or opposing force structure. SITFOR is the cell that manages the status of all the own and opposing forces in the scenario except for the ones represented by HICON and LOCON. When opposing side is also played by a part of the TA, only the parts of forces not controlled by the TA is managed by SITFOR.

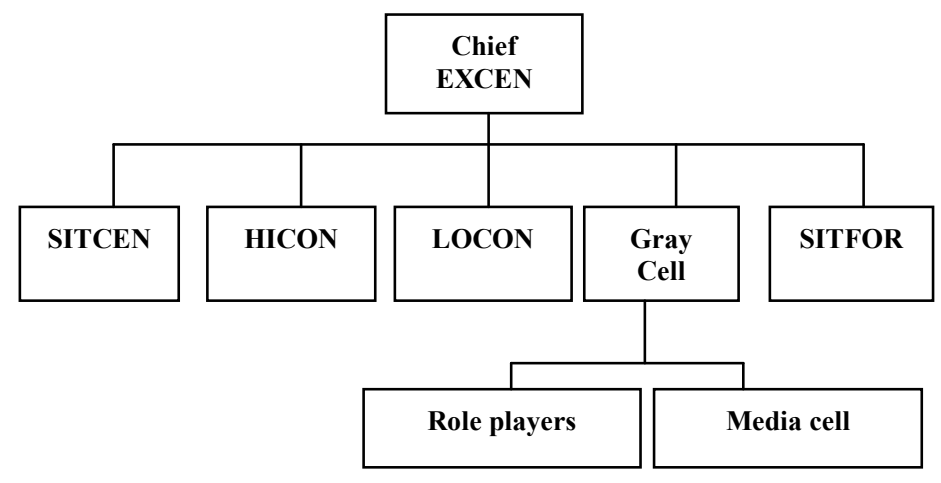

Figure 3: EXCEN model

Main Event List (MEL)/Master Incident List (MIL) team is an important team within SITCEN. In order to achieve the exercise objectives, events and incidents are designed and injections are developed according to them before the exercise. "Events are major occurrences or a sequence of related incidents, which are actions or situations that provide greater clarity to an event. An injection is the way of bringing an incident to the attention of players". MEL/MIL is not fixed, and should be modified during an exercise, i.e., dynamically scripted throughout the exercise. MEL/MIL manager closely monitors the status of every injection, and manages dynamic scripting and timings of injections. MEL/MIL team both ensures that every injection serves for a training objective (TO), and the coverage of TA, TO and the exercise duration by the injections are very well balanced and realistic.

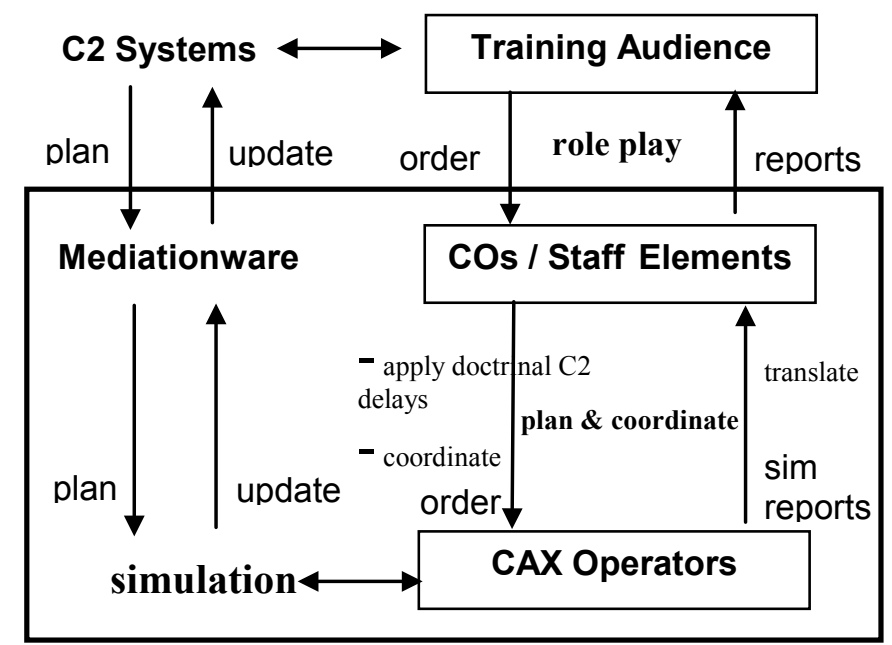

Response cells

Figure 4: Simulation and the training audience

LOCON and HICON consist of response cells (RC). The number of RC is dependent on the scenario and the TA. Each $\mathrm{RC}$ is made up of a MEL/MIL coordinator, a number of planners and a number of simulation operators. RC are the main interface between simulation and exercise as depicted in Figure 4. There are two ways for interaction between TA and simulation, and both of them are indirect. The first way is through the response cells who are acting as LOCON to the TA. TA gives an order and a response cell role plays as the subordinate unit headquarter that receives the order. Than the planners in the response cell coordinate and plan the execution of the order, and after applying some doctrinal C2 delays, they pass the plans to the CAX operators who translate them to the simulation orders. CAX operators receive both periodical and mission 
reports from the simulation system. They transfer these simulation reports to the planners in the response cell, who translates them to the reports for the TA. This interaction with the simulation system is through role play by the response cell.

The second way is automatic interaction through command and control (C2) systems. Simulation systems can interact automatically with the operational $\mathrm{C} 2$ systems of TA by using mediation-ware. For example, the air tasking orders developed in a $\mathrm{C} 2$ system can be translated into the simulation orders and directly fed into the simulation. This interaction is from the $\mathrm{C} 2$ system to the simulation. The opposite direction is also possible. For example the simulated air missions can update the recognized air picture in a $\mathrm{C} 2$ device automatically and continuously.

The bottom line is that $\mathrm{RC}$ are the main component which directly interacts with simulations in a CAX. In this construct simulation are used for the following reasons:

- Reason 1: To compute the possible outcomes, i.e., results, of the decisions made by TA.

- Reason 2: To simulate the entities and conditions not controlled by the TA or EXCON.

- Reason 3: To maintain a consistent white truth. Recognized operational pictures are derived from the white truth according to the intelligence capabilities and efforts of the sides and services.

- Reason 4: To stimulate Command and Control (C2) systems used by TA.

All these reasons are important. Although the simulation systems are designed mainly for the first two reasons, they can be used only for the last two in a completely scripted exercise, i.e., exercises where the plans of TA are not simulated, but the results of their decisions are prescript based on forecasting, and modified during the exercise according to the developing situation. This is called dynamic scripting. Of course when TA is given injections based on experience and intuition of EXCON, the risk that the injections are not coherent and realistic is higher. We call this risk as "negative training" risk because a headquarter trained with unrealistic injections may plan considering that they can achieve the same results also in a real battlefield. Therefore, verified and validated simulation systems with validated databases are important and can reduce the "negative training" risk. Realism that a simulation can provide is related to its capabilities. Multi-resolution federations concept is a both time and cost effective way to achieve constructive simulation environments with enhanced simulation capabilities.

\section{MULTI RESOLUTION FEDERATIONS AND NATO TRAINING FEDERATION}

Multi-resolution federations are federations that integrate highly aggregated and high resolution simulations into a distributed simulation system typically by using HLA. Virtual and live simulation systems can also become federates in a multi resolution federation. Therefore, it is not necessary to differentiate multi resolution federations from live virtual constructive (LVC) federations. However this differentiation is often made, i.e., LVC federations and multi resolution federations are often built separately. There are two main reasons for this:

- Virtual and live simulations have to be real time. On the other hand being able to run faster or slower than real time is a desirable feature for constructive simulations.

- Many virtual and live simulations are already federated by using DIS. There is a federation object model (FOM) called real-time platform reference (RPR) FOM designed based on DIS protocol data units (PDU). Therefore many LVC federations use RPR2 FOM. Since the latest RPR FOM does not support entity-aggregate interactions, it is not preferred for multi resolution federations.

Modular FOM approach in HLA 1516-2009 provides new techniques to create a modular FOM that can include both RPR and other modules more appropriate for multi resolution federations. There is already work to create reference architectures that can be used as both LVC and multi-resolution federations. However most of the current multi resolution federations are designed and implemented separate from LVC federations. Some examples for these implementations are joint multi resolution federation (JMRF) and joint multi resolution model (JMRM) in the US, partnership for peace simulation network (P2SN) reference architecture by NATO partner nations, KORA and SIRA federation (KOSI) in Germany, and ALLIANCE in France. Most of these federations are still maturing, and not used in a major exercise.

NATO training federation (NTF) is a multi resolution HLA federation derived from JMRM, and successfully used in a major NATO exercise first time in 2008. Initial NTF has two combat models, namely joint theatre level simulation (JTLS) (Cayirci 2002) and joint conflict and tactical simulation (JCATS). JTLS is a joint highly aggregated constructive simulation system. It best fits when the simulated units (simulation entities) are battalions, wings/air packages, i.e., multiple air crafts in an air mission, and ships (frigates, submarines, etc.) JCATS is a joint high resolution constructive simulation, where the details like a single troop can be simulated by using high resolution terrain and environmental data. It is also possible to aggregate the simulated entities into units and command them as aggregated units in JCATS. 


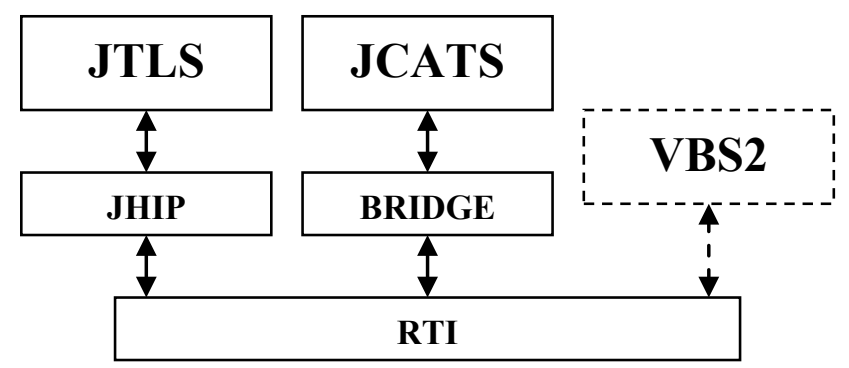

Figure 5: NATO Training federation

Currently Virtual Battle Simulation (VBS2) is also federated with JCATS through distributed interactive simulation (DIS, IEEE 1278). Making VBS2 also an HLA federate to NTF is within the plans. NTF and NATO LVC (NLVC) federations will merge into a single federation based on a refrence federation architecture recommended by a Task Group, called Modeling and Simulation Group 068 (MSG-068) of Research and Technology Agency (RTA) in NATO.

NTF is a very good example for reusability and interoperability of simulation tools. When the training audience is lower than or equal to component command (corps) level, JCATS provides better fidelity simulation. On the other hand since it is high resolution, it needs many details for operating. Therefore, it is not viable to use purely JCATS in exercises where high number of units and large areas are involved, e.g., corps and higher level exercises. JTLS is a very good match for those levels. However, many incidents of contemporary warfare and conflicts require high resolution planning also in high echelons, which is not available in JTLS. NTF connects JTLS and JCATS to close this gap. In NTF highly aggregated JTLS can be used as long as higher resolution simulation is not needed. When simulation resolution higher than the one that JTLS can provide is required, JCATS can be used. The outputs of these two simulations update the attributes of the units and entities in both simulations. C2 systems can be stimulated by one of the federates in NTF, i.e., JCATS or JTLS, or become a federate themselves.

When NTF is available, some entities are simulated by JCATS, and some units in the same theatre are simulated by JTLS. The entities in JCATS are able to interact with the units in JTLS, which means an aircraft in JCATS can fire a missile to a ship in JTLS. This creates a new question to answer: "Which entities or units should be simulated in JTLS or JCATS?" The ownership of simulated objects can also be switched between JTLS and JCATS from time to time. The mapping between the simulation requirements and simulation systems can be based on the following criteria (Cayirci 2007b):

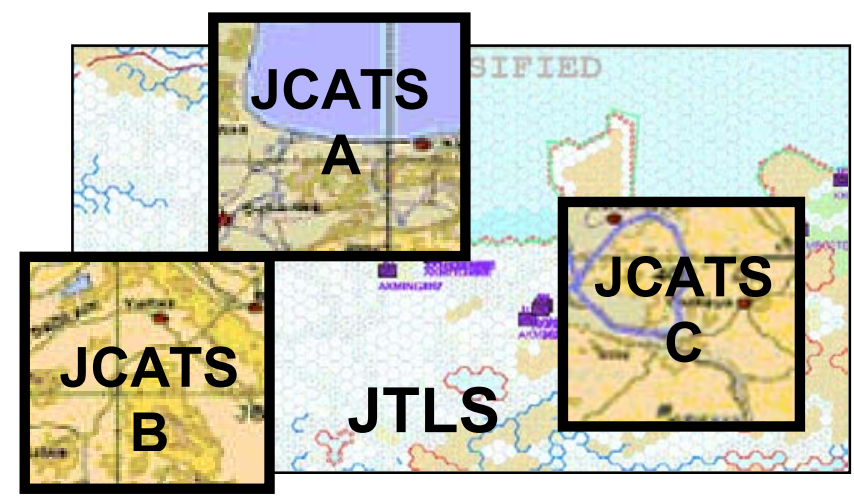

Figure 6: Geography based ownership

- Geography based sharing of the units and entities: The entities can be shared between multiple simulation systems based on geography. As shown in Figure 6 we can give the simulation responsibility of a certain area to JCATS, while the responsibility for all the other regions stays with JTLS. The JCATS may also have multiple geographic regions and change the areas from time to time. For example, Region A can be in JCATS at the beginning for a while, and then it may be returned to JTLS and Regions B and C can be taken by JCATS.

- Component based sharing of the units and entities: One of the simulations can be used to simulate everything related to a specific component (e.g., air, maritime, land, psychological operations, special operations, etc.) while the others are simulated by another simulation. For example we can simulate everything related to special operations 
component at JCATS, and all the other components at JTLS. In this case, in the same area there may be entities simulated in JCATS and units simulated in JTLS. This increases the probability that an entity in JCATS interact with a unit in JTLS.

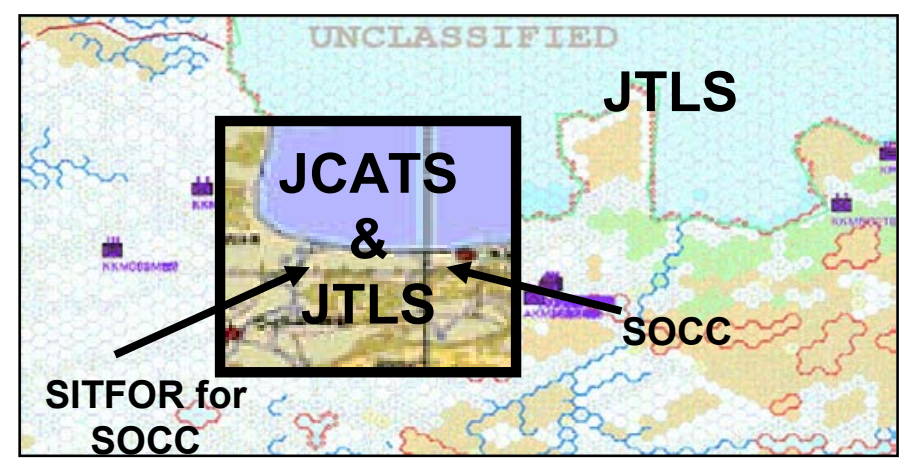

Figure 7: Component based ownership

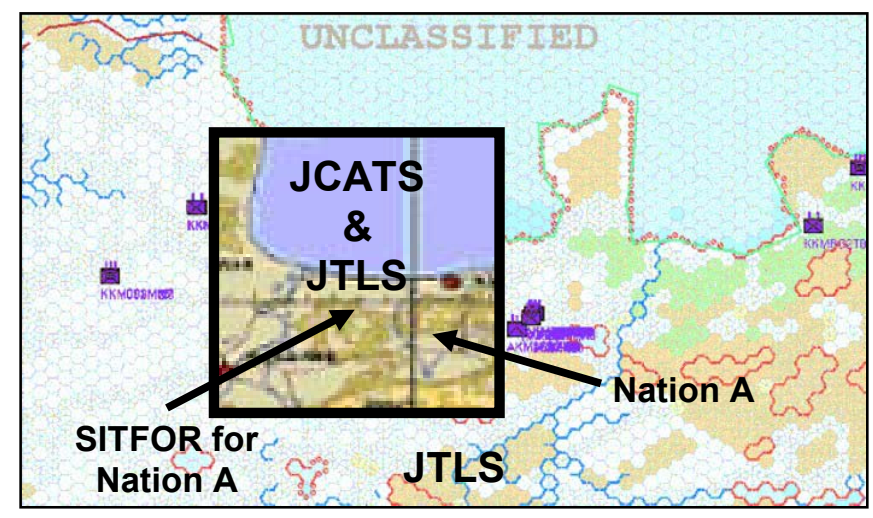

Figure 8: Nation based ownership

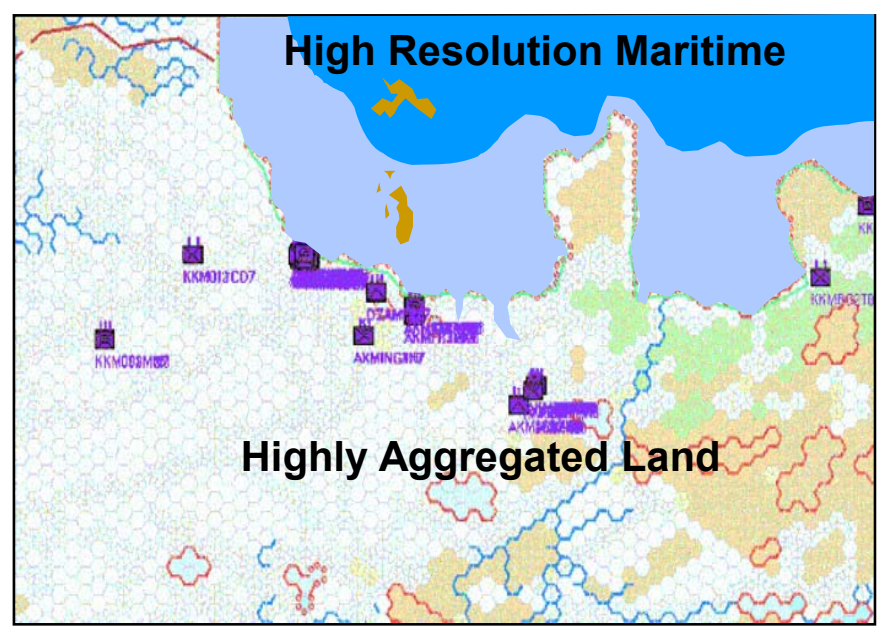

Figure 9: Service based ownership

- Nation based sharing of the units and entities: If an exercise is combined, all the entities for a nation may be simulated in a simulation system while the alliance headquarters and the forces from the other nations are simulated in another simulation. This is similar to the component based sharing. 
- Service based sharing of the units and entities: All the entities for a service can be simulated in a simulation system while all the others are in another simulation system. For example, we can use one of the simulations to simulate maritime operations, and the other for all the other ground and air operations.

- Type of operations based sharing of the units and entities: Finally operations like counter improvised explosive devices (counter IED), long distance reconnaissance, time sensitive targeting, joint personnel recovery, river crossing, amphibious, riot control can be simulated in JCATS and the others in JTLS.

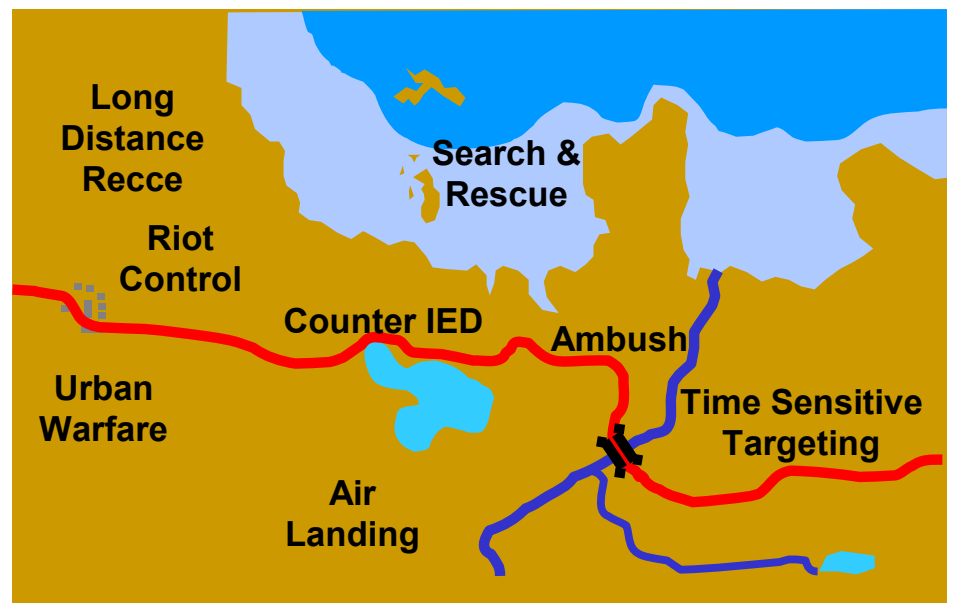

Figure 10: Operations based ownership

ADVANTAGES OF MULTI RESOLUTION FEDERATIONS

High resolution constructive simulations are very often called tactical simulations. Therefore it is argued that only highly aggregated simulations are needed for operational and higher level exercises. Although high resolution simulations fit better for the simulation requirements of tactical level exercises, they are also very useful for operational and higher level exercises because of the following two reasons:

- In exercises conducted in operational and higher level, MEL/MIL includes many incidents high resolution in nature, such as, time sensitive targeting (TST) and joint personnel recovery (JPR). In some operational level exercises these types of incidents become the majority in MEL/MIL.

- In a CAX, response cells (RC) interact with simulation, which means that the plans of RC are entered into simulation. This implies that the simulated objects are minimum two level below the lowest level TA in command hierarchy.

Before further elaborating this, we first would like to list major advantages of multi resolution federations:

- Better simulation environments, i.e., Reasons 1 and 2 in Section 2: It enables simulating also high resolution incidents more realistically in operational and higher level exercises.

- Enhanced interoperability and reusability: New capabilities can be added into a federation made up of both legacy and state of the art simulation systems. The simulation environment becomes less dependent to a specific simulation.

- Multi level training environments especially for combined operations: It supports training multiple echelons that have separate sets of training objectives at the same exercise.

- High resolution results to stimulate high resolution C2 systems: They can provide both highly aggregated or high resolution results to stimulate $\mathrm{C} 2$ systems.

- Better virtualization capabilities for operational and higher level exercises: Virtualization tools like VBS2 can become a federate to these federations. Tools like VBS2 can provide intelligence products, such as aerial pictures and video streams from unmanned aerial vehicles (UAV). Visualization of the battle field can also help the training audience to have a better insight about the possible results of their decisions.

All these are important and worth to invest. We will focus in the first and the last items in the rest of this section. We use one example incident that are very common in operational and higher level CAXs: time sensitive targeting (TST). On this example, we will explain what if there is no simulation support, there is only a highly aggregated simulation (JTLS), there is only a high resolution simulation (JCATS) and there is a multi resolution federation (NTF). 


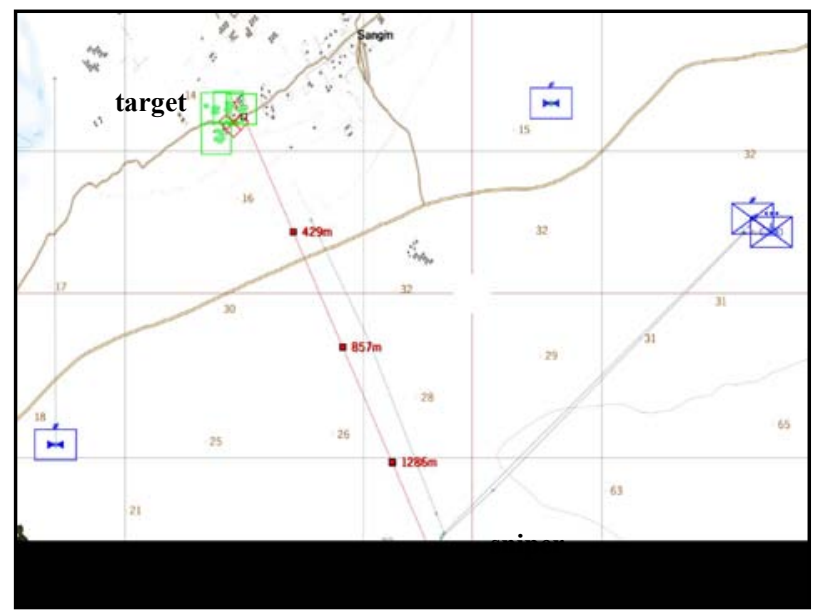

Figure 11: TST incident

In our incident an intelligence is received by a joint force command (our primary TA): "An important target is in a small village and will presumably stay there several hours." The TA assess the situation and possible courses of actions (COA). One of COAs is "to order an air strike." Since the risk of collateral casualties is high, this COA is not selected. Another COA is "to order a sniper attack." This COA seems viable. Special operations component command (SOCC), who is among the primary TA, consults that they can deploy a sniper team to the vicinity of target in time. SOCC is ordered to plan and conduct the operation. SOCC plans the deployment, which also includes deploying helicopters for evacuation and close air support in case of an escalated contact and casualties. SOCC passes the orders to its subordinates who are role players in SOCC RC. SOCC $\mathrm{RC}$ plans further and reports back that the position in the initial plans does not provide the line of sight. After that point the sniper team needs to move to a better position, which is almost $2 \mathrm{~km}$ away from the position in the initial plan. Some part of this final two km must be covered by crawling. Our four cases for this incident are compared in Table 3.

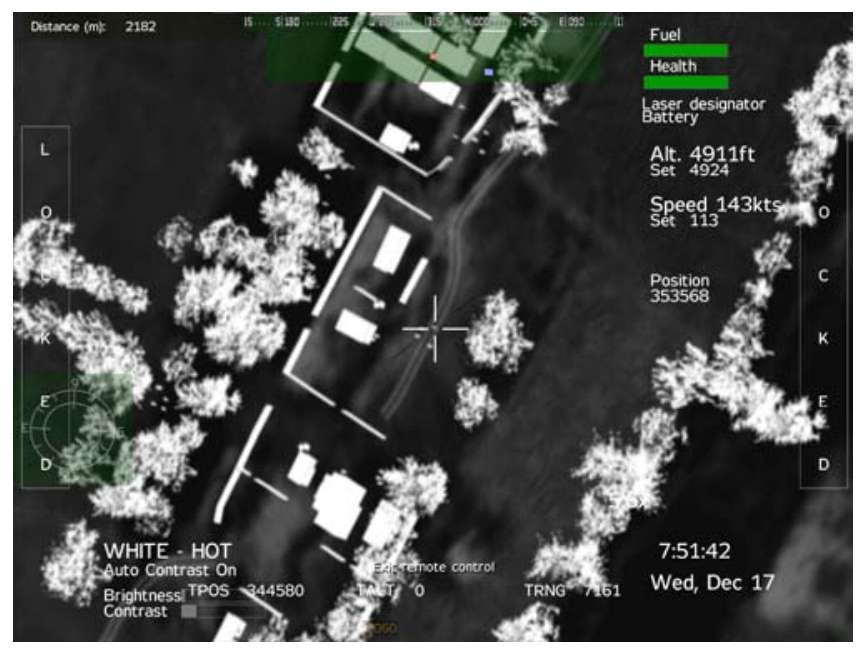

Figure 12: Live simulated video stream from a UAV simulation

Table 3 shows that the best option to simulate a TST incident during an operational level exercise is a multi-resolution federation. When a multi-resolution federation is carefully designed and complemented with effective exercise processes, it can become very cost efficient and useful in support of combined/joint operational and higher level exercises. Please note again that there is not only TST, which response cells in an operational level exercise need to deal with in high resolution. Reader should also always remember that the plans of the training audience are not entered into the simulation directly. They should first to be converted to the plans of response cells acting as the subordinates for TA. 
Table 3: Comparison of types of simulations

\begin{tabular}{|c|c|}
\hline Cases & Comments \\
\hline No Simulation & $\begin{array}{l}\text { SOCC RC and other related RC carry out all the following computation manually and reports back to } \\
\text { SOCC, i.e., TA: } \\
\text { - The availability of aircrafts, fuel, ammunition and personnel for the mission including the aircrafts for } \\
\text { close air support and evacuation. } \\
\text { - Line of sight and range assessments. } \\
\text { - Threats (sensing, detecting, engaging, probability of hit and kill in case of engagements) and their } \\
\text { impacts during the deployments through air. } \\
\text { - The time needed for air deployment. } \\
\text { - Manual updates of C } 2 \text { tools for all movement based on the air assets and their link capabilities. } \\
\text { - The time needed for the final } 2 \mathrm{~km} \text {. } \\
\text { - Threats (sensing, detecting, engaging, probability of hit and kill in case of engagements) and their } \\
\text { impacts during the final } 2 \mathrm{~km} \text {. } \\
\text { - Positive identification of target. } \\
\text { - The engagement, probability of hit and kill. } \\
\text { - Collateral casualties. } \\
\text { - Everything above for the way back. } \\
\text { No live video streams from unmanned aerial vehicles (UAV), such as the one shown in Figure } 12 \text {, can be } \\
\text { provided. }\end{array}$ \\
\hline $\begin{array}{l}\text { Highly } \\
\text { Aggregated } \\
\text { Simulation }\end{array}$ & $\begin{array}{l}\text { SOCC RC carries out all the following manually because highly aggregated simulations do not have the } \\
\text { required resolution for modeling these accurately: } \\
\text { - Line of sight and range assessments. } \\
\text { - The time needed for the final } 2 \mathrm{~km} \text {. } \\
\text { - Threats (sensing, detecting, engaging, probability of hit and kill in case of engagements) and their } \\
\text { impacts during the final } 2 \mathrm{~km} \text {. } \\
\text { - Positive identification of target. } \\
\text { - The engagement, probability of hit and kill. } \\
\text { - The collateral casualties. } \\
\text { No live video streams from UAV can be provided. }\end{array}$ \\
\hline $\begin{array}{l}\text { High } \\
\text { Resolution } \\
\text { Simulation }\end{array}$ & $\begin{array}{l}\text { High resolution simulations can model accurately and in the required level of resolution everything listed } \\
\text { above. However, the database preparation and operating the model may become too cumbersome because } \\
\text { of the overall level of the exercise. Moreover a high resolution constructive simulation system may not } \\
\text { have visualization tools for positive identification and live video streams from UAVs. }\end{array}$ \\
\hline $\begin{array}{l}\text { Multi } \\
\text { Resolution } \\
\text { Federation }\end{array}$ & $\begin{array}{l}\text { This is the right combination. Whenever higher resolution is required the simulation responsibilities is } \\
\text { passed to high resolution simulations. Video streams are provided through a visualization system like } \\
\text { VBS2. Intel products for battle damage assessment (BDA) can also be provided. Moreover the } \\
\text { architecture supports interoperability and reusability. }\end{array}$ \\
\hline
\end{tabular}

Giving examples for the other way around, i.e., why multi resolution federations are useful in tactical level training is also possible. Expeditionary forces that need long distance transportation, logistics and communications is an example for that type of use cases. Since this topic is not within the scope of this paper, we do not elaborate those examples further.

\section{CONCLUSIONS}

Several multi-resolution federations have been developed for the last decade. Most of them are not mature yet, and only few are used in a major exercise. NTF is a multi-resolution federation derived from JMRM, and used in support of a major NATO exercise in 2008. For the time being NTF is an HLA federation of JTLS and JCATS. VBS2 is also federated into NTF through DIS. NTF can cover all the required resolution levels in a major combined and joint exercise. NTF is a cost effective simulation tool, and more flexible comparing to any single simulation system or single resolution federation.

\section{REFERENCES}


Bowers, A. 2003. Multi-Resolution Modeling in the JTLS-JCATS Federation. Technical report. MITRE Cooperation. Cayirci E., Marincic D. 2009. Computer Assisted Exercises and Training: A Reference Guide. Wiley \& Sons.

Cayirci E. 2007a. Exercise Structure for Distributed Multi-resolution NATO Computer Assisted Exercises. ITEC'2007. May.

Cayirci E. 2007b. Distributed Multi-resolution Computer Assisted Exercises. NATO Modelling and Simulation Conference 2007, October.

Cayirci E. 2006. NATO Joint Warfare Center's Perspective on CAX Support Tools and Requirements. ITEC'2006. May.

Cayirci, E., Ersoy, C. 2002. Simulation of Tactical Communications Systems by Inferring Detailed Data from the Joint Theater Level Computer Aided Exercises. SCS Simulation Journal, 78: 475-484.

IEEE 2000a. IEEE Standard for Modeling and Simulation (M\&S) High Level Architecture (HLA) - Framework and Rules, Std 1516.

IEEE 2000b. IEEE Standard for Modeling and Simulation (M\&S) High Level Architecture (HLA) - Federate Interface Specification, Std 1516.1.

IEEE 2000c. IEEE Standard for Modeling and Simulation (M\&S) High Level Architecture (HLA) - Object Model Template (OMT), Std 1516.2.

Defense Modeling and Simulation Office (DMSO) 1998. High Level Architecture Interface Specification, Version 1.3NG. Washington D.C.

Reynolds, P.F., Srinivasan, S. 1997. Consistency Maintenance in Multiresolution Simulations. ACM Transactions on Modeling and Computer Simulation. 7(3): 368-392.

Surdu J.R., Poach, U.W. 2000. Simulations Technologies in the Mission Operational Environment. SCS Simulation. 74(3): 138-160.

\section{AUTHOR BIOGRAPHY}

ERDAL CAYIRCI graduated from Army Academy in 1986 and from Royal Military Academy, Sandhurst in 1989. He received his MS degree from Middle East Technical University, and a PhD from Bogazici University both in computer engineering in 1995 and 2000, respectively. He retired from the Army when he was a colonel in 2005. He was an Associate Professor at Istanbul Technical University, Yeditepe University and Naval Sciences and Engineering Institute between 2001 and 2005. Also in 2001, he was a visiting researcher for the Broadband and Wireless Networking Laboratory and a visiting lecturer at the School of Electrical and Computer Engineering, Georgia Institute of Technology. He founded Genetlab in 2005. He is currently Chief, CAX Support Branch in NATO's Joint Warfare Center in Stavanger, Norway, and also a professor in the Electrical and Computer Engineering Department of University of Stavanger. His research interests include sensor networks, mobile communications, tactical communications, and military constructive simulation. Professor Cayirci has acted as an editor of the journals IEEE Transactions on Mobile Computing, AdHoc Networks (Elsevier Science) and ACM/Kluwer Wireless Networks, and has guest edited four special issues of Computer Networks (Elsevier Science), AdHoc Networks (Elsevier Science) and Kluwer Journal on Special Topics in Mobile Networking and Applications (MONET). He received the "2002 IEEE Communications Society Best Tutorial Paper" Award for his paper titled "A Survey on Sensor Networks" published in the IEEE Communications Magazine in August 2002, the "Fikri Gayret" Award from Turkish Chief of General Staff in 2003, the "Innovation of the Year" Award from Turkish Navy in 2005 and the "Excellence" Award in ITEC 2006. He is also author of two textbooks, "Security in Wireless Ad Hoc and Sensor Networks" and "Computer Assisted Exercises: A Reference Guide," both published by John Wiley \& Sons. His email is <erdal.cayircieuis.no>. 